\title{
External Memory Orthogonal Range Reporting with Fast Updates
}

\author{
Yakov Nekrich*
}

\begin{abstract}
In this paper we describe data structures for orthogonal range reporting in external memory that support fast update operations. The query costs either match the query costs of the best previously known data structures or differ by a small multiplicative factor.
\end{abstract}

\section{Introduction}

In the orthogonal range reporting problem a set of points is stored in a data structure so that for any $d$-dimensional query range $Q=\left[a_{1}, b_{1}\right] \times \ldots \times\left[a_{d}, b_{d}\right]$ all points that belong to $Q$ can be reported. Due to its fundamental nature and its applications, the orthogonal range reporting problem was studied extensively; we refer to e.g. [10, 9, 19, 4, 8, for a small selection of important publications. In this paper we address the issue of constructing dynamic data structures that support fast update operations in the external memory model.

External memory data structures for orthogonal range reporting also received significant attention, see e.g., [16, 17, 19, 6, 1, 14, 2, 15]. We refer to [18] for the definition of the external memory model and a survey of previous results. In particular, dynamic data structures for $d=2$ dimensions are described in [16, 17, 6]. The best previously known data structure of Arge, Samoladas, and Vitter [6] uses $O\left((N / B) \log _{2} N / \log _{2} \log _{B} N\right)$ blocks of space, answers queries in $O\left(\log _{B} N+K / B\right)$ I/Os and supports updates in $O\left(\log _{B} N\left(\log _{2} N / \log _{2} \log _{B} N\right)\right)$ I/Os; in [6], the authors also show that the space usage of their data structure is optimal. Recently, the first dynamic data structure that supports queries in $O\left(\log _{B}^{2} N+K / B\right)$ I/Os in $d=3$ dimensions was described [15].

All previously described external memory data structures with optimal or almost-optimal query cost need $\Omega\left(\left(\log _{B} N \log _{2} N\right) / \log _{2} \log _{B} N\right)$ I/Os to support an insertion or a deletion of a point; see Table1. This compares unfavorably with significantly lower update costs that can be achieved by internal memory data structures. For instance, the two-dimensional data structure of Mortensen [12] supports updates in $O\left(\log _{2}^{f} N\right)$ time for any constant $f>7 / 8$. Moreover, the update costs of previously described external structures contain an $O\left(\log _{2} N\right)$ factor. Since block size $B$ can be large, achieving update cost that only depends on $\log _{B} N$ would be desirable. High cost of updates is also a drawback of the three-dimensional data structure described in [15]. Reducing the cost of update operations can be important in the dynamic scenario when the data structure must be updated frequently.

Our Results. We describe several data structures for orthogonal range reporting queries in $d=2$ dimensions that achieve lower update costs. We describe two data structures that support queries in $O\left(\log _{B} N+K / B\right)$ I/Os. These data structures support updates in $O\left(\log _{B}^{1+\varepsilon} N\right)$

\footnotetext{
${ }^{*}$ Department of Computer Science, University of Chile. Email yakov.nekrich@googlemail.com
} 


\begin{tabular}{|c|c|c|c|}
\hline Source & Query & Update & Space \\
& Cost & Cost & Usage \\
\hline$[16]$ & $O\left(\log _{B} N+\frac{k}{B}\right)$ & $O\left(\log _{B} N \log _{2} N \log _{2}^{2} B\right)$ & $O\left((N / B) \log _{2} N \log _{2} B \log _{2} \log _{2} B\right)$ \\
{$[17]$} & $O\left(\log _{B} N+\frac{k}{B}+\mathrm{ilog}(B)\right)$ & $O\left(\log _{2} N\left(\log _{B} N+\left(\log _{B}^{2} N\right) / B\right)\right)$ & $O\left((N / B) \log _{2} N\right)$ \\
{$[\underline{6}$} & $O\left(\log _{B} N+\frac{k}{B}\right)$ & $O\left(\log _{B} N \log _{2} N / \log _{2} \log _{B} N\right)$ & $O\left((N / B) \log _{2} N / \log _{2} \log _{B} N\right)$ \\
$*$ & $O\left(\log _{B} N+\frac{k}{B}\right)$ & $O\left(\log _{B}^{1+\varepsilon} N\right) \dagger$ & $O\left((N / B) \log _{2} N\right)$ \\
$*$ & $O\left(\log _{B} N+\frac{k}{B}\right)$ & $O\left(\log _{B}^{2} N\right)$ & $O\left((N / B) \log _{2} N / \log _{2} \log _{B} N\right)$ \\
$*$ & $O\left(\log _{B} N\left(\log _{2} \log _{B} N\right)^{2}+\frac{k}{B}\right)$ & $O\left(\log _{B} N\left(\log _{2} \log _{B} N\right)^{2}\right)$ & $O\left((N / B) \log _{2} N\right)$ \\
\hline
\end{tabular}

Table 1: New data structures and some previous results for $d=2$ dimensions. Our results are marked with an asterisk; $\dagger$ denotes randomized results. The result in the first row of the table can be obtained from the result in [16] using a standard technique. The function $\operatorname{ilog}(x)$ is the iterated $\log ^{*}$ function: $i \log (x)$ denotes the number of times we must apply the $\log ^{*}$ function to $x$ before the result becomes $\leq 2$, where $\log ^{*}(x)=\min \left\{t \mid \log _{2}^{(t)}(x)<2\right\}$, and $\log _{2}^{(t)}(x)$ denotes the $\log _{2}$ function repeated $t$ times.

I/Os with high probability and in $O\left(\log _{B}^{2} N\right)$ deterministic I/Os respectively. Henceforth $\varepsilon$ denotes an arbitrarily small positive constant. We also describe a data structure that uses $O\left((N / B) \log _{2} N\right)$ blocks of space, answers queries in $O\left(\log _{B} N\left(\log _{2} \log _{B} N\right)^{2}\right)$ I/Os, and supports updates in $O\left(\log _{B} N\left(\log _{2} \log _{B} N\right)^{2}\right)$ I/Os. All our results are listed in Table 1 .

Overview. The situations when the block size $B$ is small and when $B$ is not so small are handled separately. If the block size is sufficiently large, $B=\Omega\left(\log _{2}^{4} N\right)$ for an appropriate choice of constant, our construction is based on the bufferization technique. We show that a batch of $O\left(B^{1 / 4}\right)$ queries can be processed with $O\left(\log _{B} N\right)$ I/Os. Hence, we can achieve constant amortized update cost for sufficiently large $B$. In the case when $B$ is small, $B=O\left(\log _{2}^{4} N\right)$, we construct the base tree with fan-out $\log _{2}^{\varepsilon} N$ or the base tree with constant fan-out. Since $B=\operatorname{polylog}_{2}(N)$, the height of the base tree is bounded by $O\left(\log _{B} N\right)$ or $O\left(\log _{B} N \log _{2} \log _{B} N\right)$. Hence, we can reduce a two-dimensional query to a small number of simpler queries.

In section 2 we describe a data structure that supports three-sided reporting queries in $O\left(\log _{B} N+\frac{K}{B}\right)$ I/Os and updates in $O\left(\frac{1}{B^{\delta}}\right)$ I/Os if $B=\Omega\left(\log _{B}^{4} N\right)$. Henceforth $\delta$ denotes an arbitrary positive constant, such that $\delta \leq 1 / 4$. In Appendix $\mathrm{A}$, we generalize this result and obtain a data structure that supports updates in $O(1) \mathrm{I} / \mathrm{Os}$ and orthogonal range reporting queries in $O\left(\log _{B} N+\frac{K}{B}\right)$ I/Os if $B=\Omega\left(\log _{2}^{4} N\right)$. Thus if a block size is sufficiently large, there exists a data structure with optimal query cost and $O(1)$ amortized update cost. We believe that this result is of independent interest. Data structures for $B=O\left(\log _{2}^{4} N\right)$ are described in section 3 .

\section{Three-Sided Range Reporting for $B=\Omega\left(\log _{B}^{4} N\right)$}

Three-sided queries are a special case of two-dimensional orthogonal range queries. The range of a three-sided query is the product of a closed interval and a half-open interval. In this section we assume that the block size $B \geq 4 h \log _{B}^{4} N$ for a constant $h$ that will be defined later in this section. Our data structure answers three-sided queries with $O\left(\log _{B} N+K / B\right)$ I/Os and updates are supported in $O\left(1 / B^{\delta}\right)$ amortized I/Os.

Our approach is based on a combination of external priority tree [6] with buffering technique [5]. Buffering was previously used to answer searching and reporting problems in one dimension. In this section we show that buffering can be applied to three-sided range reporting problem in the case 
when $B=\Omega\left(\log _{B}^{4} N\right)$. At the beginning, we describe the external priority tree [6] data structure. Then, we show how this data structure can be modified so that a batch of $B^{\delta}$ updates can be processed in constant amortized time. Finally, we describe the procedure for reporting all points in a three-sided range $Q=[a, b] \times[c,+\infty)$.

The following Lemma is important for our construction.

Lemma $1 A$ set $S$ of $O\left(B^{1+\delta}\right)$ points can be stored in a data structure that supports three-sided reporting queries in $O(K / B) I / O s$, where $K$ is the number of points in the answer; this data structure can be constructed with $O\left(B^{\delta}\right) I / O s$.

Proof: We can use the data structure of Lemma 1 from [6].

External Priority Tree. Leaves of the external priority tree contain the $x$-coordinates of points in sorted order. Every leaf contains $\Theta(B)$ points and each internal node has $\Theta\left(B^{\delta}\right)$ children. We assume throughout this section that the height of an external priority tree is bounded by $h \log _{B} N$. The range $r n g(v)$ of a node $v$ is the interval bounded by the minimal and the maximal coordinates stored in its leaves; we say that a point $p$ belongs to (the range of) a node $v$ if its $x$-coordinate belongs to the range of $v$. Each node is associated with a set $S(v),|S(v)|=\Theta(B)$, defined as follows. Let $L(v)$ denote the set of all points that belong to the range of $v$. The set $S(v)$ contains $B$ points with largest $y$-coordinates among all points in $L(v)$ that do not belong to any set $S(w)$, where $w$ is an ancestor of $v$. Thus external priority tree is a modification of the priority tree with node degree $B^{O(1)}$, such that each node contains $\Theta(B)$ points.

The data structure $F(v)$ contains points from $\cup S\left(v_{i}\right)$ for all children $v_{i}$ of $v$. By Lemma 1 , $F(v)$ supports three-sided queries in $O(1)$ I/O operations. Using $F(v)$, we can answer three-sided queries in $O\left(\log _{B} N+K / B\right) \mathrm{I} /$ Os; the search procedure is described in [6].

Supporting Insertions and Deletions. Now we describe a data structure that supports both insertions and deletions. We will show below how a batch of inserted or deleted points can be processed efficiently. The main idea is to maintain buffers with inserted and deleted points in all internal nodes. The buffer $D(v), v \in T$, contains points that are stored in descendants of $v$ and must be deleted. The buffer $I(v), v \in T$, contains points that must be inserted into sets $S(u)$ for a descendant $u$ of $v$. A buffer can contain up to $B^{3 \delta}$ elements. When a buffer $I(v)$ or $D(v)$ is full, we flush it into the children $v_{j}$ of $v$; all sets $I\left(v_{j}\right), D\left(v_{j}\right)$, and $S\left(v_{j}\right)$ are updated accordingly.

Definitions of $S(v)$ and $F(v)$ are slightly modified for the dynamic structure. Every set $S(v)$ contains at most $2 B$ points. If $S(v)$ contains less than $B / 2$ points than $S\left(v_{i}\right)=\emptyset$ for each child $v_{i}$ of $v$. The data structure $F(v)$ contains all points from $S\left(v_{j}\right) \cup I\left(v_{j}\right)$ for all children $v_{j}$ of $v$. We store an additional data structure $R(v)$ in each internal node. $R(v)$ contains all points from $\cup S\left(v_{i}\right)$ for all children $v_{i}$ of $v . R(v)$ can be constructed in $O\left(B^{\delta}\right) \mathrm{I} /$ Os; we can obtain $B^{3 \delta}$ points with highest $y$-coordinates stored in $R(v)$ in $O(1) \mathrm{I} / O s$. Implementation of $R(v)$ is very similar to implementation of $F(v)$; details will be given in the full version.

Suppose that all points from the set $\mathcal{D},|\mathcal{D}|=O\left(B^{\delta}\right)$, must be deleted. We remove all points from $\mathcal{D} \cap S\left(v_{r}\right)$ and $\mathcal{D} \cap I\left(v_{r}\right)$ from $S\left(v_{r}\right)$ and $I\left(v_{r}\right)$ respectively. We set $D\left(v_{r}\right)=D\left(v_{r}\right) \cup\left(\mathcal{D} \backslash S\left(v_{r}\right)\right)$. When $D(v)$ for an internal node $v$ is full, $|D(v)|=B^{3 \delta}$, we distribute the points of $D(v)$ among the children $v_{j}$ of $v$. Let $D_{j}(v)$ be the points of $D(v)$ that belong to the range of $v_{j}$ and update $S\left(v_{j}\right)$, $D\left(v_{j}\right)$ as described above: We remove all points from $D_{j}(v) \cap S\left(v_{j}\right)$ and $D_{j}(v) \cap I\left(v_{j}\right)$ from $S\left(v_{j}\right)$ and $I\left(v_{j}\right)$ respectively. All points of $D_{j}(v) \backslash S\left(v_{j}\right)$ are inserted into $D\left(v_{j}\right)$. Finally, we update $F(v)$ and $R(v)$. 
We can insert a batch $\mathcal{I}$ of $B^{\delta}$ points using a similar procedure. Initially, all points from $\mathcal{I}$ are inserted into buffer $I\left(v_{r}\right)$ or $S\left(v_{r}\right)$ and points of $\mathcal{I} \cap D\left(v_{r}\right)$ are removed from $D\left(v_{r}\right)$. Let $S^{\prime}\left(v_{r}\right)=S\left(v_{r}\right) \cup \mathcal{I}$ and let $S^{\prime \prime}\left(v_{r}\right)$ be the set of $B$ points with highest $y$-coordinates in $S^{\prime}\left(v_{r}\right)$. We set $S\left(v_{r}\right)=S^{\prime \prime}\left(v_{r}\right)$ and $I\left(v_{r}\right)=I\left(v_{r}\right) \cup\left(S^{\prime}\left(v_{r}\right) \backslash S^{\prime \prime}\left(v_{r}\right)\right)$. When the buffer $I(v)$ in an internal node $v$ is full, $|I(v)| \geq B^{3 \delta}$, we update the sets $S\left(v_{j}\right)$ and $I\left(v_{j}\right)$ in the children $v_{j}$ of $v$. Let $I_{j}(v)$ be the set of points in $I(v)$ that belong to the range of $v_{j}$. Let $S^{\prime}\left(v_{j}\right)=S\left(v_{j}\right) \cup I_{j}(v)$ and let $S^{\prime \prime}\left(v_{j}\right)$ be the set of $B$ points with the highest $y$-coordinates in $S^{\prime}\left(v_{j}\right)$. We set $S\left(v_{j}\right)=S^{\prime \prime}\left(v_{j}\right)$, $D\left(v_{j}\right)=D\left(v_{j}\right) \backslash\left(D\left(v_{j}\right) \cap I_{j}(v)\right)$, and $I\left(v_{j}\right)=I\left(v_{j}\right) \cup\left(S^{\prime}\left(v_{j}\right) \backslash S^{\prime \prime}\left(v_{j}\right)\right)$. The data structures $F(v)$ and $R(v)$ are updated accordingly.

When a buffer $I(v)$ is full, we can re-build all $I\left(v_{j}\right), D\left(v_{j}\right), S\left(v_{j}\right)$ and the data structures $F(v), R(v)$ in $O\left(B^{\delta}\right)$ I/Os. Each inserted point is inserted in $O\left(\log _{B} N\right)$ buffers $I(v)$. Hence, an amortized cost of re-building secondary data structures caused by an insertion is $O\left(B^{\delta} \log _{B} N / B^{3 \delta}\right)=O\left(1 / B^{\delta}\right)$. The cost of a deletion can be analyzed in the same way.

We also take care that the number of points stored in sets $S(u)$ is not too small. Suppose that the number of points in some $S(w)$ is smaller than $B / 2$ when $D$ (parent $(w)$ ) is emptied. If $w$ is a leaf or $S\left(w_{j}\right)=\emptyset$ for all children $w_{j}$, we do not need to rebuild $S(w)$. Otherwise, we move some points from $S\left(w_{j}\right)$ into $S(w)$. Using the data structure $R(w)$, we identify $B-|S(w)|$ points with the highest $y$-coordinates in $\cup_{j} S\left(w_{j}\right)$. These points are removed from $R(w), F(w), S\left(w_{j}\right)$ and inserted into $S(w)$. We also update $F(\operatorname{parent}(w))$ and $R(\operatorname{parent}(w))$. For every child $w_{j}$ of $w$, we recursively call the same procedure. The total cost of updating all data structures in a node is $O\left(B^{1-3 \delta}\right)$. Using standard analysis, we can show that maintaining the size of $S(w)$ incurs an amortized cost $O\left(1 / B^{\delta}\right)$.

Besides that, we should take care that each leaf contains $x$-coordinates of at most $2 B$ points. To maintain this invariant, the external priority tree is implemented as a WBB-tree [7]. The branching parameter of our WBB-tree equals to $B^{\delta}$ and the leaf parameter equals to $B$. When the total number of points stored in all descendants of a node $u$ equals to $2 B^{\ell \delta} \cdot B$, we split the node $u$ into $u^{\prime}$ and $u^{\prime \prime}$. A node on level $\ell$ is split at most once after a series of $\Theta\left(B^{\ell \delta} \cdot B\right)$ insertions. When a node is split, we assign each element of $S(u), I(u)$, and $D(u)$ to the corresponding set in $u^{\prime}$ or $u^{\prime \prime}$. As a result, either $S\left(u^{\prime}\right)$ or $S\left(u^{\prime \prime}\right)$ may contain less than $B / 2$ elements. In this case, we move the points from descendants of $u^{\prime}$ into $u^{\prime}$ (from descendants of $u^{\prime \prime}$ into $u^{\prime \prime}$ ) as described above. The total amortized cost of splitting a node is $O\left(1 / B^{\delta}\right)$.

Answering Queries. Consider a query $Q=[a, b] \times[c,+\infty)$. Let $\pi$ denote the set of all nodes that lie on the path from the root to $l_{a}$ or on the path from the root to $l_{b}$, where $l_{a}$ and $l_{b}$ are the leaves that contain $a$ and $b$ respectively. Then all points inside the range $Q$ are stored in sets $S(v)$ or $I(v)$, where the node $v$ belongs to $\pi$ or $v$ is a descendant of a node that belongs to $\pi$. Two following facts play crucial role in the reporting procedure.

Fact 1 Let $w$ be an ancestor of a node $v$. For any $p \in S(v)$ and $p^{\prime} \in S(w), p . y<p^{\prime}$.y. For any $p \in I(v)$ and $p^{\prime} \in S(w), p . y<p^{\prime} . y$.

Fact 2 Suppose that a point $p \in S(v)$ is deleted from $S$ (but $p$ is not deleted from $S(v)$ yet). Then, $p$ belongs to a set $D(w)$ for an ancestor $w$ of $v$.

We set the value of the constant $h$ so that the height of $T$ does not exceed $h \log _{B} N$. As follows from the Fact 2, the total number of deleted points in $S(v)$ is bounded by $h \cdot B^{3 \delta} \log _{B} N \leq B / 4$. Let $D E L(v)=\cup_{w=\operatorname{anc}(v)}\left(D(w) \backslash \cup_{w^{\prime}=\operatorname{anc}(w)} I\left(w^{\prime}\right)\right)$, where anc $(u)$ denotes an ancestor of a node $u$. 
To wit, $D E L(v)$ is the set of all points $p$, such that $p$ belongs to some set $D(w)$ for an ancestor $w$ of $v$, but $p$ does not belong to any $I\left(w^{\prime}\right)$ for an ancestor $w^{\prime}$ of $w$. By Fact 2 all points in $S(v) \cup I(v)$ that are already deleted from the data structure belong to $D E L(v)$. If the set $D E L(v)$ is known, then $D E L\left(v_{i}\right)$ for a child $v_{i}$ of $v$ can be constructed in $O(1)$ I/Os. Therefore we can construct $D E L(v)$ for all $v \in \pi$ in $O\left(\log _{B} N\right)$ I/Os.

We can output all points that belong to $Q$ using the following procedure. Let $\pi_{1}$ be the path from $l_{a}$ to the lowest common ancestor $v_{l}$ of $l_{a}$ and $l_{b}$. Let $\pi_{2}$ be the path from $l_{b}$ to $v_{l}$. First, we examine all nodes $v \in \pi$ and report all points $p \in(S(v) \cup I(v)) \backslash D E L(v)$ that belong to $Q$; this can be done with $O\left(\log _{B} N\right)$ I/Os. All other points in $S \cap Q$ are stored in a set $S_{u}$ where $u$ is a descendant of some $v \in \pi_{1} \cup \pi_{2}$ or $u$ is a descendant of $v_{l}$.

Consider a node $v \in \pi_{2}$, such that $v \neq v_{l}$; we will show how points in $S(u) \cap Q$ for all descendants $u$ of $v$ can be reported. Suppose that the child $v_{i}$ of $v$ also belongs to $\pi_{2}$ and $r n g\left(v_{i-1}\right)=\left[a^{\prime}, b^{\prime}\right]$. Let $Q_{v}=\left[a, b^{\prime}\right] \times[c,+\infty)$. For a point $p$ stored in a descendant $u$ of $v$ such that $u \notin \pi_{2}, p$ belongs to $Q$ if and only if $p$ belongs to $Q_{v}$. All $p \in Q_{v} \cap S(u)$ are reported as follows. Initially we set $u=v$. We identify all points stored in $S\left(u_{i}\right) \cap Q_{v}$ or $I\left(u_{i}\right) \cap Q_{v}$ for some child $u_{i}$ of $u$ using the data structure $F(u)$. Then, we process the resulting list of points and remove all points that belong to $D E L(u)$. Finally, we identify all non-leaf children $u_{i}$ of $u$ such that at least $B / 2$ points from $S\left(u_{i}\right)$ are reported. We visit every such $u_{i}$, compute $D E L\left(u_{i}\right)$, and recursively call the same procedure in $u_{i}$.

Our procedure reports all points in $L(v) \cap Q_{v}$. Suppose that we visited a node $u$, but the child $u_{j}$ of $u$ was not visited. All points from $\left(S\left(u_{j}\right) \cup I\left(u_{j}\right)\right) \cap Q$ were reported when the node $u$ was visited. Since $S\left(u_{j}\right)$ contains at least $B / 2$ points, at least one point $p_{j} \in S\left(u_{j}\right)$ does not belong to $Q$. The $x$-coordinate of $p_{j}$ belongs to $[a, b]$; hence, the $y$-coordinate of $p_{j}$ is smaller than $c$. By Fact 1, $y$-coordinates of all points stored in $S(\nu) \cup I(\nu)$ for any descendant $\nu$ of $u_{j}$ are smaller than $c$. Hence, all points $p \in S(\nu) \cup I(\nu)$ are not relevant for our query.

The search procedure spends $O(1)$ I/Os in every visited node (ignoring the cost of reporting points). Let $K_{v}$ be the total number of reported points in $L(v) \cap Q_{v}$. A node $u$ is visited if at least $B / 4$ points from $S(u)$ were reported. Thus we can charge at least $B / 4$ points for every visited node. We can conclude that the search procedure spends $O\left(K_{v} / B\right) \mathrm{I} / \mathrm{Os}$ in the descendants of $v$. Descendants of nodes $v \in \pi_{1}, v \neq v_{l}$, and descendants of $v_{l}$ can be processed with a similar procedure. Therefore the total query cost is $O\left(\log _{B} N+K / B\right)$. We obtain the following result.

Lemma 2 Suppose that $B^{\delta} \geq 4 h \log _{B} N$ for a constant $h$ defined above and some $\delta \leq 1 / 4$. Then there exists a data structure that uses $O(N / B)$ blocks of space and answers three-sided reporting queries in $O\left(\log _{B} N+K / B\right) I / O s$. The amortized cost of inserting or deleting a point is $O\left(1 / B^{\delta}\right)$.

A similar approach can be used to construct the data structure for general two-dimensional range reporting queries.

Lemma 3 Suppose that $B^{\delta} \geq 4 h_{1} \log _{2} N$ for a constant $h_{1}$ defined in Appendix $A$ and some $\delta \leq 1 / 4$. Then there is a data structure that uses $O\left((N / B) \log _{2} N / \log _{2} \log _{B} N\right)$ blocks of space and answers two-dimensional orthogonal range reporting queries in $O\left(\log _{B} N+K / B\right) I / O s$. The amortized cost of inserting or deleting a point is $O(1)$.

Our data structure uses the bufferization technique, but some additional ideas are also needed to retain the $O\left(\log _{B} N+K / B\right)$ query cost and achieve the optimal space usage. We provide the details in the Appendix A. 


\section{Two-Dimensional Range Reporting for small $B$}

It remains to consider the case when the block size $B$ is small. In this section we assume that $B=O\left(\log _{2}^{4} N\right)$ and describe several data structures for this case.

Reduction to Three-Sided Queries. We use the base structure that is similar to structures in [17, 6]. We construct the base tree $T$ with fan-out $\rho=\Theta\left(\log _{2}^{\varepsilon} N\right)$ on the set of $x$-coordinates. In every node $v$ of $T$ we store the data structures that support three-sided queries $[a,+\infty) \times[c, d]$ and $(-\infty, b] \times[c, d]$. The data structures for three-sided queries are implemented using the external priority search tree [6], so that the query and update costs are $O\left(\log _{B} N+K / B\right)$ and $O\left(\log _{B} N\right)$. In every node $v$, we also store a data structure that supports the following queries: for any $c<d$ and for any $1 \leq i \leq j \leq \rho$, we can report all points $p$, such that $p . y \in[c, d]$ and $p$ is stored in the child $v_{f}$ of $v, i \leq f \leq j$. In [6] the authors describe a linear space data structure that supports such queries in $O(\rho+K / B) \mathrm{I} /$ Os and updates in $O\left(\log _{B} N\right) \mathrm{I} /$ Os.

To answer a query $[a, b] \times[c, d]$, we identify the lowest node $v$, such that $[a, b] \subset r n g(v)$. Suppose that $[a, b]$ intersects with $r n g\left(v_{l}\right), r n g\left(v_{l+1}\right), \ldots r n g\left(v_{r}\right)$. We answer three-sided queries $[a,+\infty) \times[c, d]$ and $(-\infty, b] \times[c, d]$ on data structures for nodes $v_{l}$ and $v_{r}$ respectively. Then, we report all points $p$ with $c \leq p . y \leq d$ stored in the nodes $v_{l+1}, \ldots, v_{r-1}$. Since $\rho=O\left(\log ^{\varepsilon} N\right)=$ $O\left(\log _{B} N\right)$, the total query cost is $O\left(\log _{B} N+\frac{K}{B}\right)$.

Since each point is stored in $O\left(\log _{2} N / \log _{2} \log _{2} N\right)$ data structures, the space usage of structure is $O\left((N / B) \log _{2} N / \log _{2} \log _{2} N\right)=O\left((N / B) \log _{2} N / \log _{2} \log _{B} N\right)$ because $B=O\left(\log _{2}^{4} N\right)$. The update cost is $O\left(\log _{B} N\left(\log _{2} N / \log _{2} \log _{2} N\right)\right)=O\left(\log _{B}^{2} N\right)$. Combining this result with Lemma 3 , we obtain the following Theorem

Theorem 1 There is a data structure that uses $O\left((N / B) \log _{2} N / \log _{2} \log _{B} N\right)$ blocks of space and answers orthogonal range reporting queries in two dimensions in $O\left(\log _{B} N+\frac{K}{B}\right) I / O$ operations. Updates are supported in $O\left(\log _{B}^{2} N\right)$ amortized I/Os.

Reduction to One-Dimensional Queries. We can obtain further results by reducing a twodimensional query to a number of one-dimensional queries. We construct a standard range tree with constant fan-out on the $x$-coordinates of points. All points that belong to a range of a node $v$ are stored in $v$. For any interval $[a, b]$, we can find $O\left(\log _{2} N\right)$ nodes $u^{i}$, such that $p . x \in[a, b]$ if and only if $p$ is stored in a node $u^{i}$. Hence, all points in the query range $Q=[a, b] \times[c, d]$ can be reported by answering a one-dimensional query $Q_{y}=[c, d]$ in $O\left(\log _{2} N\right)$ nodes $u^{1}, \ldots, u^{t}$ of the range tree. Using the fractional cascading technique, we can find the predecessor $d\left(u^{i}\right)$ of $d$ and the successor $c\left(u^{i}\right)$ of $c$ in all nodes $u^{i}$ in $O\left(\log _{2} N \log _{2} \log _{2} N\right)$ time; we refer to e.g., 11 for details. When we know $c\left(u^{t}\right)$ and $d\left(u^{t}\right)$ we can report all elements stored in the node $u^{t}$ in $O(K / B) \mathrm{I} /$ Os. Hence, the query cost is $O\left(\log _{2} N \log _{2} \log _{2} N+K / B\right)=O\left(\log _{B} N\left(\log _{2} \log _{B} N\right)^{2}+K / B\right)$. Each point is stored in $O\left(\log _{2} N\right)$ secondary data structures. As described in [11, the range tree augmented with fractional cascading data structures can be updated in $O\left(\log _{2} N\left(\log _{2} \log _{2} N\right)\right)=O\left(\log _{B} N\left(\log _{2} \log _{B} N\right)^{2}\right)$ time; hence, an update requires $O\left(\log _{B} N\left(\log _{2} \log _{B} N\right)^{2}\right)$ I/O operations.

Theorem 2 There exists a data structure that uses $O\left((N / B) \log _{2} N\right)$ blocks of space and answers orthogonal range reporting queries in two dimensions in $O\left(\log _{B} N\left(\log _{2} \log _{B} N\right)^{2}+\frac{K}{B}\right) I / O$ operations. Updates are supported in $O\left(\log _{B} N\left(\log _{2} \log _{B} N\right)^{2}\right)$ amortized I/Os.

Range Trees with $B^{O(1)}$ Fan-Out. Let $\varepsilon^{\prime}=\varepsilon / 10$. If points have integer coordinates, we can reduce the query cost by constructing a range tree with fan-out $B^{\varepsilon^{\prime}}$. For every node $v$ and every pair 
of indexes $i \leq j$, where $v_{i}, v_{j}$ are the children of $v$, all points that belong to the children $v_{i}, \ldots, v_{j}$ of $v$ belong to a list $L_{i j}(v)$. A data structure $E_{i j}(v)$ supports one-dimensional one-reporting queries on a set of integers. That is, $E_{i j}(v)$ enables us to find for any interval $[c, d]$ some point $p \in L_{i j}(v)$ such that $p . y \in[c, d]$, if such $p$ exists and if all points have integer coordinates. As described in [13], we can implement $E_{i j}(v)$ so that queries are supported in $O(1)$ time and updates are supported in $O\left(\log ^{\varepsilon^{\prime}}\right)$ randomized time. Using $E_{i j}(v)$, it is straightforward to report all $p \in L_{i j}(v)$ with $p . y \in[c, d]$ in $O(K / B)$ I/Os. Consider a query $Q=[a, b] \times[c, d]$. We can find in $O\left(\log _{B} N\right) \mathrm{I} / \mathrm{O}$ operations $O\left(\log _{B} N\right)$ nodes $u^{t}$ and ranges $\left[i_{t}, j_{t}\right]$, so that the $x$-coordinate of a point $p$ belongs to $[a, b]$ if and only if $p$ is stored in some list $L_{i_{t} j_{t}}\left(u^{t}\right)$. Hence, all points in $Q$ can be reported by reporting all points in $L_{i_{t} j_{t}}\left(u^{t}\right)$ whose $y$-coordinates belong to $[c, d]$. The total query cost is $O(\log N / \log \log N)=O\left(\log _{B} N\right)$. However, the space usage is $O\left((N / B) \log _{2}^{1+8 \varepsilon^{\prime}} N\right)$ because each point is stored in $O\left(B^{2 \varepsilon^{\prime}} \log _{B} N\right)=O\left(\log _{2}^{1+8 \varepsilon^{\prime}} N\right)$ lists $L_{i j}(v)$. We can reduce the space usage if only parts of lists $L_{i j}(v)$ stored explicitly.

Let $L(v)$ denote the list of all points that belong to a node $v$ sorted by their $y$-coordinates. We divide $L(v)$ into groups of points $G_{s}(v), s=O\left(|L(v)| / B^{1+2 \varepsilon^{\prime}}\right)$, so that each $G_{s}(v)$ contains at least $B^{1+2 \varepsilon^{\prime}} / 2$ and at most $2 B^{1+2 \varepsilon^{\prime}}$ points. Instead of $L_{i j}(v)$, we store the list $\widetilde{L}_{i j}(v)$. The main idea of our space saving method is that we need to store points of $G_{s}(v)$ in the list $\widetilde{L}_{i j}(v)$ only in the case when $G_{s}(v)$ contains a few points from $L_{i j}(v)$. Otherwise all relevant points can be found by querying the set $G_{s}(v)$ provided that $\widetilde{L}_{i j}(v)$ contains a pointer to $G_{s}(v)$. Points and pointers are stored in each list $\widetilde{L}_{i j}(v)$ according to the following rules. If $\left|L_{i j}(v) \cap G_{s}(v)\right| \leq B / 2$, the list $\widetilde{L}_{i j}(v)$ contains all points from $L_{i j}(v) \cap G_{s}(v)$. If $L_{i j}(v) \cap G_{s}(v) \geq 2 B$, the list $\widetilde{L}_{i j}(v)$ contains a pointer $p t r_{s}$ to $G_{s}(v)$. We also store the minimal and maximal $y$-coordinates of points in $L_{i j}(v) \cap G_{s}(v)$ with each pointer to $G_{s}(v)$ from $\widetilde{L}_{i j}(v)$. If $B / 2<\left|L_{i j}(v) \cap G_{s}(v)\right|<2 B, \widetilde{L}_{i j}(v)$ contains either a pointer to $G_{s}(v)$ or all points from $L_{i j}(v) \cap G_{s}(v)$.

Instead of $E_{i j}(v)$, we will use several other auxiliary data structures. A data structure $\widetilde{E}_{i j}(v)$ contains information about elements of $\widetilde{L}_{i j}(v)$. For each point $p \in \widetilde{L}_{i j}(v)$ we store $p . y$ in $\widetilde{E}_{i j}(v)$; for every pointer $p \operatorname{tr}_{s}, \widetilde{E}_{i j}(v)$ contains both the minimal and the maximal $y$-coordinate associated with $\operatorname{ptr}_{s}$. A data structure $E(v)$ contains the $y$-coordinates of all points in $L(v)$. Both $E(v)$ and all $E_{i j}(v)$ support one-reporting queries as described above. A data structure $H_{s}(v)$ supports orthogonal range reporting queries on $G_{s}(v)$. Using the data structure described in Lemma 1 of [6], we can answer three-sided reporting queries in $O(K / B)$ I/Os using $O\left(\left|G_{s}(v)\right| / B\right)$ blocks of space. Using the standard approach, we can extend this result to a data structure that uses $O\left(\left(\left|G_{s}(v)\right| \log _{2} B\right) / B\right)$ blocks and answers queries in $O(K / B) \mathrm{I} /$ Os.

Now we show how we can report all points $p \in L_{i j}(v)$ with $p . y \in[c, d]$ without storing $L_{i j}(v)$. We can find an element $e$ of $\widetilde{L}_{i j}(v)$ with $y$-coordinate in $[c, d]$. Suppose that such $e$ is found. Then, we traverse the list $\widetilde{L}_{i j}(v)$ in $+y$ direction starting at $e$ until a point $p$ with $p . y>d$ or a pointer to $G_{s}(v)$ with the minimal $y$-coordinate larger than $d$ is found. We also traverse $\widetilde{L}_{i j}(v)$ in $-y$ direction until a point $p$ with $p . y<c$ or a pointer to $G_{s}(v)$ with the maximal $y$-coordinate smaller than $c$ is found. For every pointer in the traversed portion of $\widetilde{L}_{i j}(v)$, we visit the corresponding group $G_{s}(v)$ and report all points $p \in G_{s}(v) \cap L_{i j}(v)$ with $p . y \in[c, d]$. All relevant points in $G_{s}(v)$ can be reported in $O\left(K_{s} / B\right) \mathrm{I} /$ Os using the data structure $H_{s}(v)$; here $K_{s}$ denotes the number of points reported by $H_{s}(v)$. By definition of $\widetilde{L}_{i j}(v)$, a set $G_{s}(v) \cap L_{i j}(v)$ contains at least $B / 2$ points if there is a pointer ptr from $\widetilde{L}_{i j}(v)$ to $G_{s}(v)$. Unless $p t r$ is the first or the last element in the traversed portion of $\widetilde{L}_{i j}(v), G_{s}(v)$ contains $B$ points from $[a, b] \times[c, d]$. Since $B$ consecutive 
elements of the list $\widetilde{L}_{i j}(v)$ contain either $B$ points or at least one pointer to a group $G_{s}(v)$, the total cost of reporting all points in $L_{i j}(v)$ with $p . y \in[c, d]$ is $O(1+K / B)$.

Now we consider the situation when there is no $e \in \widetilde{E}_{j}(v)$, such that $e \in[c, d]$. In this case $L_{i j}(v)$ may contain some points from the range $Q$ only if all points $p \in L(v)$ with $p . y \in[c, d]$ belong to one group $G_{s}(v)$. Using $E(v)$, we search for a point $p_{s} \in L(v)$ such that $p_{s} . y \in[c, d]$. If there is no such $p_{s}$, then $L(v) \cap Q=\emptyset$. Otherwise $p_{s} \in G_{s}(v)$ and we can report all points in $Q \cap G_{s}(v)$ in $O(1+K / B) \mathrm{I} /$ Os using $H_{s}(v)$. We need to visit $O\left(\log _{B} N\right)$ nodes of the range tree to answer the query; hence, the total query cost is $O\left(\log _{B} N+K / B\right) \mathrm{I} /$ Os.

Since the lists $L_{i j}(v)$ are not stored, the space usage is reduced to $O\left((N / B) \log _{2} N\right)$ : Each list $\widetilde{L}_{i j}(v)$ contains less than $B$ points and at most one pointer for each group $G_{s}(v)$. Since $L(v)$ is divided into $O\left(|L(v)| / B^{1+2 \varepsilon^{\prime}}\right)$ groups, the total size of all $L_{i j}(v)$ is $O(|L(v)|)$. All data structures $H_{s}(v)$ for all groups $G_{s}(v)$ use $O\left(\left(|L(v)| \log _{2} B\right) / B\right)$ blocks of space. Each point belongs to $O\left(\log _{B} N\right)$ nodes; therefore the total space usage is $O\left((N / B) \log _{2} N\right)$.

When a new point $p$ is inserted, we must insert it into $O\left(\log _{B} N\right)$ lists $L(v)$. Suppose that $p$ is inserted into $G_{s}(v)$ in a node $v$. We insert $p$ into $H_{s}(v)$ in $O\left(\log _{2} B\right) \mathrm{I} / \mathrm{Os} ; p$ is also inserted into up to $B^{2 \varepsilon^{\prime}}=O\left(\log _{2}^{8 \varepsilon^{\prime}} N\right)$ lists $\widetilde{L}_{i j}(v)$. The one-dimensional reporting data structure for $\widetilde{L}_{i j}(v)$ supports updates in $O\left(\log _{2}^{\varepsilon^{\prime}} N\right)$ I/Os; hence, the total cost of inserting a point is $O\left(\log _{2}^{9 \varepsilon^{\prime}} N\right)$. For each pair $i \leq j$, we check whether the number of points in $L_{i j}(v) \cap G_{s}(v)$ equals to $2 B$. Although the list $L_{i j}(v)$ is not stored, we can estimate the number of points in $L_{i j}(v) \cap G_{s}(v)$ by a query to the data structure $H_{s}(v)$. If $\left|L_{i j}(v) \cap G_{s}(v)\right|=2 B$, we remove all points of $\widetilde{L}_{i j}(v) \cap G_{s}(v)$ from $L_{i j}(v)$ and insert a pointer to $G_{s}(v)$ into $\widetilde{L}_{i j}(v)$. Points in a list $\widetilde{L}_{i j}(v)$ are replaced with a pointer to a group $G_{s}(v)$ at most once for a sequence of $\Theta(B)$ insertions into $G_{s}(v)$. Hence, the amortized cost of updating $\widetilde{L}_{i j}(v)$ because the number of points from $L_{i j}(v)$ in a group exceeds $2 B$ is $O\left(\log _{2}^{\varepsilon^{\prime}} N\right)$ I/Os. Each insertion affects $O\left(\log _{2}^{8 \varepsilon^{\prime}} N\right)$ lists $L_{i j}(v)$. If the number of points in $G_{s}(v)$ equals $2 B \log _{2}^{1+2 \varepsilon^{\prime}} N$, we split the group $G_{s}(v)$ into $G_{1}(v)$ and $G_{2}(v)$ of $B \log _{2}^{1+2 \varepsilon^{\prime}} N$ points each. Since up to $B$ elements can be inserted and deleted into every list $\widetilde{L}_{i j}(v)$, the amortized cost incurred by splitting a group is $O\left(\log _{2}^{9 \varepsilon^{\prime}} N\right)$. Thus the total cost of inserting a point into data structures associated with a node $v$ is $O\left(\log _{2}^{9 \varepsilon^{\prime}} N\right)$ I/Os. Since a new point is inserted into $O\left(\log _{2} N / \log _{2} \log _{2} N\right)$ nodes of the range tree, the total cost of an insertion is $O\left(\log _{2}^{1+9 \varepsilon^{\prime}} N / \log _{2} B\right)=O\left(\log _{B}^{1+\varepsilon} N\right)$. Deletions are processed in a symmetric way.

Combining this result with Lemma 4, we obtain the following Theorem

Theorem 3 Suppose that point coordinates are integers. There exists a data structure that uses $\mathrm{O}\left((\mathrm{N} / \mathrm{B}) \log _{2} \mathrm{~N}\right)$ blocks of space and answers orthogonal range reporting queries in two dimensions in $O\left(\log _{B} N+\frac{K}{B}\right) I / O$ operations. Updates are supported in $O\left(\log _{B}^{1+\varepsilon} N\right)$ amortized I/Os w.h.p. for any $\varepsilon>0$.

\section{References}

[1] P. Afshani, On Dominance Reporting in 3D, Proc. ESA 2008, 41-51.

[2] P. Afshani, L. Arge, K. D. Larsen, Orthogonal Range Reporting in Three and Higher Dimensions, Proc.FOCS 2009, 149-158.

[3] A. Aggarwal, J. S. Vitter, The Input/Output Complexity of Sorting and Related Problems, Communications of the ACM 31(9), 1116-1127 (1988). 
[4] S. Alstrup, G. S. Brodal, T. Rauhe, New Data Structures for Orthogonal Range Searching, Proc. FOCS 2000, 198-207.

[5] L. Arge, The Buffer Tree: A Technique for Designing Batched External Data Structures, Algorithmica 37, 1-24 (2003).

[6] L. Arge, V. Samoladas, J. S. Vitter, On Two-Dimensional Indexability and Optimal Range Search Indexing, Proc. PODS 1999, 346-357.

[7] L. Arge, J. S. Vitter, Optimal External Memory Interval Management, SIAM J. Comput. 32(6), 1488-1508 (2003).

[8] T. Chan, Persistent Predecessor Search and Orthogonal Point Location on the Word RAM, Proc. SODA 2011, .

[9] B. Chazelle, A Functional Approach to Data Structures and its Use in Multidimensional Searching, SIAM J. on Computing, 17, 427-462 (1988).

[10] H. N. Gabow, J. L. Bentley, R. E. Tarjan, Scaling and Related Techniques for Geometry Problems, Proc. STOC 1984, 135-143.

[11] K. Mehlhorn, S. Näher, Dynamic Fractional Cascading, Algorithmica 5, 215-241 (1990).

[12] C. W. Mortensen, Fully Dynamic Orthogonal Range Reporting on RAM, SIAM J. Computing 35(6), 1494-1525 (2006).

[13] C. W. Mortensen, R. Pagh, M. Patrascu, On Dynamic Range Reporting in One Dimension. Proc. STOC 2005, 104-111.

[14] Y. Nekrich, I/O-Efficient Point Location in a Set of Rectangles. Proc. LATIN 2008, 687-698.

[15] Y. Nekrich, Dynamic Range Reporting in External Memory, Proc. ISAAC 2010, 25-36.

[16] S. Ramaswamy, S. Subramanian, Path Caching: A Technique for Optimal External Searching, Proc. PODS 1994, 25-35.

[17] S. Subramanian, S. Ramaswamy, The P-range Tree: A New Data Structure for Range Searching in Secondary Memory, Proc. SODA 1995, 378-387.

[18] J. S. Vitter, External Memory Algorithms and Data Structures: Dealing with Massive Data, ACM Computing Surveys, 33(2), 209-271 (2001).

[19] D. E. Vengroff, J. S. Vitter, Efficient 3-D Range Searching in External Memory, Proc. STOC 1996, 192-201.

\section{Appendix A. Two-Dimensional Range Reporting for $B=\Omega\left(\log _{2}^{4} N\right)$}

We maintain a constant fan-out tree $T$ on the set of $x$-coordinates of all points. An internal node of $T$ has at most eight children. A point $p$ belongs to an internal node $v$, if its $x$-coordinate is stored in a leaf descendant of $v$. We assume that the height of $T$ is bounded by $h_{1} \log _{2} N$. Each node $v$ 
contains two secondary data structures that support three-sided queries of the form $[a,+\infty) \times[c, d]$ and $(-\infty, b] \times[c, d]$ respectively; both data structures contain all points that belong to $v$. We also store all points that belong to $v$ in a B-tree sorted by their $y$-coordinates, so that all points with $y$-coordinates in an interval $[c, d]$ can be reported. The data structures for three-sided queries are implemented as described in Lemma 2, We implement the B-tree using the result of [5], so that updates are supported in $O\left(1 / B^{\delta}\right) \mathrm{I} /$ Os. Hence, updates on the secondary data structures are supported in $O\left(1 / B^{\delta}\right) \mathrm{I} / \mathrm{Os}$.

We say that a node $v$ of $T$ is special if the depth of $v$ is divisible by $\left\lceil\delta \log _{2} B / 3\right\rceil$. To facilitate the query processing, buffers with inserted and deleted elements will be stored in the special nodes only. A node $u$ is a direct special descendant of $v$ if $u$ is a special node, $u$ is a descendant of $v$, and there is no other special node $u^{\prime}$ on the path from $v$ to $u$. We denote by $\operatorname{desc}(v)$ the set of direct special descendants of a node $v$. The set of nodes $\operatorname{subset}(v)$ consists of the node $v$ and all nodes $w$, such that $w$ is a descendant of $v$ and $w$ is an ancestor of some node $u \in \operatorname{desc}(v)$. In other words, every node $w$ on a path from $v$ to one of its direct special descendants belongs to subset $(v)$; the node $v$ also belongs to $\operatorname{subset}(v)$.

Let $\bar{I}(v)$ and $\bar{D}(v)$ denote the buffers of inserted and deleted points stored in a node $v \in T$. When a point is inserted, we add it to the buffer $\bar{I}\left(v_{R}\right)$, where $v_{R}$ is the root of $T$. When a buffer $I(v)$ contains at least $B^{2 \delta}$ elements, we visit every node $w \in \operatorname{subset}(v)$ and insert all points $p \in I(v) \cap r n g(w)$ into the secondary data structures of a node $w$. Then, we examine all nodes $u \in \operatorname{desc}(v)$. For every $u \in \operatorname{desc}(v)$, we insert all points $p \in \bar{I}(v) \cap r n g(u)$ into $\bar{I}(u)$ and remove all points $p \in(\bar{I}(v) \cap r n g(u)) \cap \bar{D}(u)$ from $\bar{D}(u)$. Finally, we set $I(v)=\emptyset$.

The total number of nodes in $\operatorname{subset}(v)$ and $\operatorname{desc}(v)$ is $O\left(B^{\delta}\right)$. Since each point is inserted into $O\left(\log _{2} B\right)$ data structures and the total number of points is $O\left(B^{2 \delta}\right)$, all data structures in subset $(v)$ can be updated in $O\left(B^{2 \delta} \log _{2} B / B^{\delta}\right)=O\left(B^{\delta} \log _{2} B\right)$ I/Os. We can also update the buffers $\bar{I}(u)$ and $\bar{D}(u)$ for each $u \in \operatorname{desc}(v)$ in $O(1)$ I/Os. Hence, a buffer $I(v)$ can be emptied in $O\left(B^{\delta} \log _{2} B\right)$ I/Os. Since a buffer $I(v)$ is emptied once after $\Theta\left(B^{2 \delta}\right)$ points were inserted into $I(v)$, the amortized cost of an insertion into $I(v)$ is $O\left(\log _{2} B / B^{\delta}\right)$. An insertion of a point $p$ into the data structure leads to insertions of $p$ into $O\left(\log _{B} N\right)$ buffers $I(v)$. Hence, the amortized cost of inserting a point $p$ is $O\left(\log _{2} N / B^{\delta}\right)=O(1)$. Deletions can be processed with a simmetric procedure.

Consider a query $Q=[a, b] \times[c, d]$. We identify the node $v$ of $T$ such that $[a, b] \subset r n g(v)$, but $[a, b] \not \subset r n g\left(v_{i}\right)$ for any child $v_{i}$ of $v$. Suppose that $[a, b]$ intersects with $r n g\left(v_{l}\right), \ldots, r n g\left(v_{r}\right)$ where $1 \leq l \leq r \leq 4$. All points $p \in S \cap Q$ are stored in the secondary structures of nodes $v_{l}, \ldots, v_{r}$ or in buffers of the special ancestors of $v$ (possibly including the node $v$ itself). We start by constructing sets $I N S(v)$ and $D E L(v)$. The set $I N S(v)$ contains all points $p$ such that $p \in \bar{I}(w)$ for an ancestor $u$ of $v$, but $p \notin \bar{D}\left(u^{\prime}\right)$ for an ancestor $u^{\prime}$ of $u$. The set $D E L(v)$ contains all points $p$ such that $p \in \bar{D}(w)$ for an ancestor $u$ of $v$, but $p \notin \bar{I}\left(u^{\prime}\right)$ for an ancestor $u^{\prime}$ of $u$. Only $O\left(\log _{B} N\right)$ ancestors of $v$ are special nodes and every buffer stored in a special node contains at most $B^{2 \delta}$ points. Hence, both $I N S(v)$ and $D E L(v)$ can be constructed in $O\left(\log _{B} N\right)$ I/Os and contain $h_{1} \cdot B^{2 \delta} \log _{B} N \leq B / 4$ points. We output all points of $p \in I N S(v) \cap Q$ in $O(1) \mathrm{I} /$ Os. Let $\mathcal{V}$ be the list of all points $p$, such that $p$ belongs to $Q$ and $p$ is stored in a child of $v$. The list $\mathcal{V}$ can be generated as follows. First, we answer three-sided queries $[a,+\infty) \times[c, d]$ and $(-\infty, b] \times[c, d]$ on data structures for nodes $v_{l}$ and $v_{r}$ respectively. Then, we identify all points $p$ stored in a node $v_{j}, l<j<r$, such that $c \leq p . y \leq d$. When the list $\mathcal{V}$ is constructed, we traverse $\mathcal{V}$ and output all points of $\mathcal{V}$ that do not belong to the set $D E L$. The list $\mathcal{V}$ can be generated and traversed in $O\left(\log _{B} N+\frac{|\mathcal{V}|}{B}\right) \mathrm{I} / \mathrm{Os}$. Since the total number of points in the answer is $K \geq|\mathcal{V}|-B / 4$, all points of $\mathcal{V} \backslash D E L$ can be identified 
and reported in $O\left(\log _{B} N+\frac{|K|}{B}\right)$ I/Os.

Our result is summed up in the following lemma

Lemma 4 Suppose that $B^{\delta} \geq 4 \log _{2} N$ for a constant $\delta \leq 1 / 4$. Then there exists a data structure that uses $\mathrm{O}\left((\mathrm{N} / \mathrm{B}) \log _{2} \mathrm{~N}\right)$ blocks of space and answers two-dimensional orthogonal range reporting queries in $O\left(\log _{B} N+K / B\right) I / O s$. The amortized cost of inserting or deleting a point is $O(1)$.

We can slightly improve the space usage by increasing the fan-out of the base tree. Our construction is the same as above, but every internal node has $\Theta\left(\log _{B} N\right)$ children. We also store an additional data structure $H(v)$ in every internal node $v$ of $T$. For any $l<r$ and any $c \leq d, H(v)$ enables us to efficiently report all points $p$, such that $p . y \in[c, d]$ and $p$ is also stored in a child $v_{j}$ of $v$ for $l<j<r$. The data structure $H(v)$ is described below.

Let $L(v)$ denote the list of all points that belong to $v$. Let $Y(v)$ be the set that contains $y$ coordinates of all points in $L(v)$. For every point $p \in L\left(v_{l}\right)$ and for all children $v_{l}$ of $v, H(v)$ contains a "point" $\tau(p)=\left(p . y, \operatorname{succ}\left(p . y, Y\left(v_{l}\right)\right)\right)$. For a query $c, H(v)$ returns all points $p \in L(v)$ such that $\tau(p) \in(-\infty, c] \times[c,+\infty)$. In other words, we can report all points $p \in L(v)$ such that $p . y \leq c$ and $\operatorname{succ}\left(p . y, Y\left(v_{l}\right) \geq c\right.$. An answer to query contains $O\left(\log _{B} N\right)$ points; at most one point for each child $v_{l}$. Using Lemma 2, $H(v)$ supports queries and updates in $O\left(\log _{B} N+K\right)=O\left(\log _{B} N\right) \mathrm{I} / \mathrm{Os}$ and updates in $O\left(1 / B^{\delta}\right)$ amortized I/Os respectively.

We can report all points $p \in L\left(v_{j}\right)$ such that $p . y \in[c, d]$ and $l<j<r$ as follows. Using $H(v)$, we search for all points $p$, such that $p . y \leq c$ and $\operatorname{succ}\left(p . y, Y\left(v_{l}\right) \geq c\right.$. For every found $p, l<j<r$, we traverse the list $L\left(v_{j}\right)$ and report all points that follow $p$ until a point $p^{\prime}, p^{\prime} . y>d$, is found. The total query cost is $O\left(\log _{B} N+K / B\right)$.

The global data structure supports insertions and deletions of points in the same way as shown in Lemma 4. The query answering procedure is also very similar to the procedure in the proof of Lemma 4. We identify the node $v$ of $T$ such that $[a, b] \subset r n g(v)$, but $[a, b] \not \subset r n g\left(v_{i}\right)$ for any child $v_{i}$ of $v$. We also find the children $v_{l}, \ldots, v_{r}$ of $v$ such that $[a, b]$ intersects with $r n g\left(v_{l}\right), \ldots, r n g\left(v_{r}\right)$. The sets $I N S(v), D E L(v)$, and the list $\mathcal{V}$ can be generated as described above. The only difference is that we identify all points $p$ stored in nodes $v_{j}, l<j<r$, such that $c \leq p$.y $\leq d$ using the data structure $H(v)$. 